P812 LEVERAGING PEAK DAYS/TIME AT SPOTS TO IMPROVE KEY POPULATION HIV PROGRAMS - SIZE ESTIMATION STUDY OF 10 STATES IN NIGERIA

${ }^{1}$ Greg Ashefor*, ${ }^{1}$ Adaoha Anosike, ${ }^{1}$ Idoteyin Ezirim, 'Olutosin Adebanjo, ${ }^{2}$ Chukumebuka Ejeckam, ${ }^{3}$ Kalada Green, ${ }^{4}$ Wole Fajemisin, ${ }^{4}$ Godpower Omoregie. ${ }^{1}$ National Agency for the Control of AIDS, Research Monitoring and Evaluation, Abuja, Nigeria; ${ }^{2}$ University of Manitoba, Abuja, Nigeria; ${ }^{3}$ Centre for Global Public Health - Nigeria, Abuja, Nigeria; ${ }^{4}$ Society for Family Health, Monitoring and Evaluation, Abuja, Nigeria

10.1136/sextrans-2019-sti.860

Background In Nigeria, HIV prevalence is high amongst key populations (KP). Mode of transmission study revealed that $38 \%$ of new-infections in Nigeria are attributable to KPs. To plan KPs interventions, programmatic mapping was conducted to provide insight on locations where KPs are found and peak day/time during which the highest number of KPs visit these locations

Methods Three KP groups (FSW, PWID, and MSM) were mapped in 10 states. Programmatic mapping which involved two sequential data collection steps known as level one [L1] and level two [L2] was adopted During L1, information was collected from key informants (KIs) on geographic locations/ spots where KPs congregate, characteristics of spots and estimate of KPs found there. During L2, KI interviews were conducted at spots identified in L1. In L2 interviews primary KIs (FSWs, IDUs, MSM,) validated information collected during L1.

Results 32,556 KI interviews were conducted in L1. 16,563 spots (8,877 FSW spots, 4,349 MSM spot, 3,837 PWID spots) were identified in 10 states. Peak days are when at least $80 \%$ of KPs visit spots. For FSW spots, 8 states have peak days on Saturday and Sunday while in 2 states (Taraba, Gombe states) it is only on Sunday. For MSM spots, Abia, Imo Anambra, Enugu, Kaduna, Oyo states have peak days on Saturday and Sunday while Edo, Kano and Taraba states have peak day on Sunday. PWID spot peak days are on Saturday and Sunday in Abia, Edo, Enugu, Oyo, Anambra, Imo, Kaduna and Kano states while Gombe and Taraba have peak day on Sunday. On peak days, peak time is mostly from $5 \mathrm{pm}$ to 12 midnight in all states for FSW and MSM. For PWID, peak time is mostly from $5 \mathrm{pm}$ to $9 \mathrm{pm}$.

Conclusion With these results, Nigeria can design and implement HIV programs targeting KPs around days and times to achieve maximum reach.

Disclosure No significant relationships.

\section{P813 COMMUNITY-BASED HEALTH SERVICES DELIVERY AMONG KEY AND PRIORITY POPULATIONS - A CASE STUDY IN UGANDA}

${ }^{1}$ Geoffrey Mujisha*, ${ }^{2}$ Kenneth Kulu. ${ }^{1}$ Most At-Risk Populations (MARPs) Network Limited, Strategy, Kamwokya, Kampala, Uganda; ${ }^{2}$ Most At-Risk Populations (MARPs) Network Limited, Knowledge Management, Kamwokya, Kampala, Uganda

\subsection{6/sextrans-2019-sti.861}

Background Freedom and diversity drop in centres (FaDDiCs), also known as safe spaces are gaining momentum in many countries. The initial FaDDiCs in Uganda were co-located within clinics. While clinic-based FaDDiCs offered some tailored services, it was only after the introduction of community-based FaDDiCs that remarkable uptake of services was registered among hidden key populations; particularly sex workers (SWs), men who have sex with men (MSM), transgender (TG) and people who use and inject drugs (PWUID). MNL established $22 \mathrm{FaDDiCs}$ to provide community-responsive services, offer psychosocial support, health information, condoms, lubricants, referrals and outreach sites for other biomedical interventions.

Methods

FaDDiCs Selection MNL identified sites for FaDDiCs after peer-led planning and preparatory activities including; hotspot mapping and feasibility assessment of each proposed location for safety, security, accessibility and population targeted. Tools and techniques: MNL equipped each $\mathrm{FaDDiC}$ with an edutainment set, condom dispensers, tools for data collection and reporting on $\mathrm{FaDDiC}$ operations. The attendant peer educators and FaDDiC managers were immediately enrolled into a quarterly training program to equip them with skills and techniques for community-facility linkage. Service Delivery Mode: FaDDiCs open daily and receive and make referrals through a clear networking and linkage pathway.

Results 22 FaDDiCs were selected in Kampala (15) and Wakiso (7) districts; 6 for SWs, 6 for MSM, 5 for TG, 1 for PWUID, 1 for LBQ, 1 for truckers, 1 for fisher-folk and 1 for prison population. In 15 months, FaDDiCs received 6343 clients, provided condoms to 4988 clients, lubricants to 4018 clients and referred 1264 clients to health facilities. FaDDiCs also attracted other interventions such as the roll out of PrEP and HIV self-testing.

Conclusion FaDDiCs offer an alternative service delivery avenue to marginalised populations, decongest the over stretched public health facilities and offer an opportunity for customised care to key populations.

Disclosure No significant relationships.

\section{P814 COMPREHENSIVE HEALTHCARE INTERVENTIONS AT MEKELLE UNIVERSITY STI AND ART CLINICS FOR KEY POPULATION, NORTHERN ETHIOPIA}

${ }^{1}$ Tesfay Gebrehiwot*, ${ }^{2}$ Kibrom Tadesse. 'Mekelle University, School of Public Health, Department of Health Systems, Mekelle, Ethiopia; ${ }^{2}$ Mekelle University HIV Care and Treatment Project, Mekelle, Ethiopia

\subsection{6/sextrans-2019-sti.862}

Background Reducing the burden of sexual transmitting infections (STIs) among key population group has a considerable role in prevention of HIV to the general population. Ethiopia is one of the sub Saharan African countries with high burden of HIV epidemic. As a response, Mekelle University provided comprehensive health care to prevent STIs/HIV prevention through its confidential STI clinics among 6550 female sex workers on selected hot spot areas.

Methods This study highlighted the experience of MU STI clinics on provision of health care interventions to the key population. Data were obtained from a register of female sex workers recorded for purpose of service provision at confidential STI clinics in Mekelle, Adigrat and Mekoni Ethiopia from April 2015 to September 30, 2018. A descriptive analysis of the services delivered to patients was performed.

Results The prevalence of STIs among the female sex workers increased from $4.5 \%$ in 2015 to $6.8 \%$ in FY 2018. Of the clients counseled and tested for HIV at the three confidential STI clinics; 77/4826 (1.59\%), 42/3843 (1.09\%) and 48/2116 (2.27\%) at Mekelle, Adigrat and Meknoi were found to be reactive for HIV respectively. The possible reasons for the 